\title{
Abstract Title: Why Should I Take My Medicine? A Review Of ED Visits For Seizures.
}

Authors:

John Logan Reed ${ }^{1,2,3}$

Thomas E. Gutwein ${ }^{1,2,4,5,6}$

Author Department and School Affiliation:

${ }^{1}$ Indiana University School of Medicine-Fort Wayne; ${ }^{2}$ Student Education and Research Fellowship Program; ${ }^{3}$ Indiana University Medical Student Program for Research and Scholarship; ${ }^{4}$ Parkview Health Emergency Department; ${ }^{5}$ Parkview Mirro Center for Research and Innovation; ${ }^{6}$ Fort Wayne Medical Education Program

Abstract Body:

\section{Study hypothesis}

The costs of anti-epileptic medication (AED) and poor care coordination result in increased Emergency Department (ED) visits for seizure events and produce both direct and indirect economic burdens on patients with frequent seizures.

\section{Methods}

A retrospective chart review study of ED visits with chief concern of seizure from two hospitals over a two-year period was performed; resulting in 152 visits recorded in this study. Data collected included demographic information, relevant seizure or past medical history (PMH), diagnostics performed in the ED, and the admission status of the patient along with the total charges per encounter. Data was analyzed descriptively and with logistic regression analysis.

\section{Results}

The results yielded by this study were generally in-line with the results of similar studies, indicating a higher relative rate of ED seizure visits for males, people of Black race, and infant and toddler populations. A high proportion of Medicaid/Medicare coverage and indiscernible employment status for most patients were also noted. Observed differences in average cost among patients with and without epilepsy and male versus female patients were not shown to be statistically significant. However, the increased likelihood of admission with increasing age was shown to be significant with an average age difference between admitted and discharged populations of approximately 10 years $(p=$ $0.003)$.

\section{Conclusions}


The data provided here is not sufficient to examine the complex relationship between seizures, epilepsy, and costs among various other patient factors. Further study is necessary to minimize direct and indirect costs of seizures.

\section{Acknowledgements}

This project was funded, in part, with support from the Indiana Clinical and Translational Sciences Institute funded, in part by UL1TR002529 from the National Institutes of Health. The content is solely the responsibility of the authors and does not necessarily represent the official views of the National Institutes of Health. 